\title{
An Efficient Deterministic-Stochastic Model of the Human Body Exposed to ELF Electric Field
}

\author{
Anna Šušnjara and Dragan Poljak \\ Department of Electronics, University of Split, 21000 Split, Croatia \\ Correspondence should be addressed to Dragan Poljak; dpoljak@fesb.hr
}

Received 14 August 2016; Accepted 18 September 2016

Academic Editor: Yuan Yao

Copyright (C) 2016 A. Šušnjara and D. Poljak. This is an open access article distributed under the Creative Commons Attribution License, which permits unrestricted use, distribution, and reproduction in any medium, provided the original work is properly cited.

\begin{abstract}
The paper deals with the deterministic-stochastic model of the human body represented as cylindrical antenna illuminated by a low frequency electric field. Both analytical and numerical (Galerkin-Bubnov scheme of Boundary Element Method) deterministic solutions of the problem are outlined. This contribution introduces the new perspective of the problem: the variability inherent to input parameters, such as the height of the body, the shape of the body, and the conductivity of body tissue, is propagated to the output of interest (induced axial current). The stochastic approach is based on the stochastic collocation (SC) method. Computational examples show the mean trend of both analytically and numerically computed axial current with the confidence margins for different set of input random variables. The results point out the possibility of improving the efficiency in calculation of basic restriction parameter values in electromagnetic dosimetry.
\end{abstract}

\section{Introduction}

The exposure of the human body to electromagnetic fields has always been a kind of controversy, particularly in the last two decades. During this period, a number of papers dealing with various models of the human body have been published. The interaction of the human body with electromagnetic field can be analyzed separately for low and high frequencies, respectively, due to different coupling mechanisms and related biological effects. Due to the absence of resonance effects at low frequencies (very long wavelengths, e.g., $6000 \mathrm{~km}$ at $50 \mathrm{~Hz}$ ), the thermal effects are negligible, while the nonthermal effects could possibly have severe effects on membrane cells $[1,2]$. On the other hand, in high frequency range where the body dimensions are comparable to external field wavelength and resonances become significant, thermal effects are dominant $[3,4]$. In the last few decades, various epidemiological surveys can be found regarding this subject. The study of the adverse health effects of electromagnetic fields to humans is still a hot topic as there are no clear correlations between the EM field exposure and diseases, such as cancer.
As it has been already stressed in $[1,2,5]$, the key to understanding the coupling of low frequency (LF) fields with the human body is the knowledge of the induced current inside the human body. When the body is exposed to tangential electric field, the induced current consists only of axial component while circulating component of current induced by magnetic field is small and thus negligible $[1,2]$. The approaches to tackling this problem can be generally classified as analytical and numerical. Once the current induced inside the human body is determined, it is straightforward to calculate the current density, electric field, and other characteristic parameters [5].

In this paper, the cylindrical antenna model of the human body exposed to extremely low frequency (ELF) electromagnetic field is considered. King and Sandler solved the problem analytically in [6] proposing parasitic antenna model of the human body and Gandhi and Chen developed realistic human body model and solved it using FDTD [7], while Poljak and Rashed dealt with the boundary element model of the human body and solved it in frequency domain by using the Galerkin-Bubnov scheme [5]. Numerical calculation of 
induced current inside the human body exposed to ELF electric field has also been reported in [8-10].

The nature of the parameters encountered in dosimetry studies is uncertain. The influence of their variability has to be taken into account in order to obtain full knowledge about the induced current or electric field inside the human body. Also, in order to convey good validation among the different computational and experimental models, it is necessary to develop a stochastic model that provides a statistical distribution of recommended limits [11]. The present paper aims to implement stochastic modelling to account for the variability of calculated induced current inside the human body due to the uncertain nature of input parameters required for the current assessment, such as the height of a person, the shape of the body, and the corresponding body conductivity, which is the dominant electric parameter of the body at ELF. The aim of the paper is to combine modern stochastic techniques, namely, stochastic collocation (SC) with the existing deterministic model for the induced current calculation, and thus provide a new tool for more accurate determination of the body model.

In this paper, SC technique is combined with the analytical solution proposed by King $[1,2]$ and numerical solution proposed by Poljak and Rashed [5]. The emphasis of this work is put on the novel deterministic-stochastic approach; therefore, rather simplified model of the human body is chosen, but it is worth noting that due to increased computational capability the human body models have become more anatomically realistic. A detailed review of realistic male and female models is given in [12].

The paper is organized as follows: in Section 2, an overview of the theoretical antenna model of the human body exposed to ELF fields is presented. The overview of analytical and numerical solution, respectively, of this model is given. In Section 3, the statistical background is explained, and, in Section 4, some illustrative numerical results are presented. The obtained results are compared with the basic restriction (BR) levels given by the ICNIRP guidelines [13-15]. Finally, in Section 5, some conclusions are drawn.

\section{Deterministic Cylindrical Model of the Human Body}

2.1. Formulation. Human is assumed to be vertically positioned on the perfectly conductive ground (PEC) and exposed to incident ELF field. As it has been reported in $[1,2$, 5], the human body exposed to ELF field can be represented by an imperfectly conducting thick cylinder whose length corresponds to the height of the body and its radius is calculated according to the mean width of the human body ( $L$ and $a$, resp., in Figure 1). It is common to use muscle tissue in homogenous models of the human body which is the reason to use the conductivity of muscle tissue as the average conductivity of the equivalent cylindrical antenna $[3,4]$.

The man is standing on the ground with arms alongside the body and barefoot, thus neglecting the capacity between the soles of the feet and their image in the earth; that is, the body is well grounded. The mean values for height, radius, and conductivity are $1.75 \mathrm{~m}, 0.14 \mathrm{~m}$, and $0.5 \mathrm{~S} / \mathrm{m}$, respectively $[1,2,5]$.

Total axial current induced in the body is governed by the Pocklington integrodifferential equation that can be derived from the continuity condition for the tangential electric field components [5]:

$$
E_{z}^{\mathrm{inc}}+E_{z}^{\mathrm{sct}}=I(z) Z_{L}(z)
$$

where $E_{z}^{\mathrm{inc}}$ is the incident field, $E_{z}^{\text {sct }}$ is scattered field due to the presence of imperfectly conductive cylinder, $I(z)$ is induced axial current, and $Z_{L}(z)$ is the impedance per unit length of the finitely conductive cylinder.

Start from the curl Maxwell equation:

$$
\nabla \times \vec{E}=-j \omega \mu \vec{H}
$$

and take into account the definition of magnetic vector potential:

$$
\vec{H}=\frac{1}{\mu} \nabla \times \vec{A} .
$$

The scattered electric field can be expressed as follows [5]:

$$
E_{z}^{\mathrm{sct}}=-\frac{j \omega}{k^{2}}\left(\frac{\partial^{2} A_{z}}{\partial z^{2}}+k^{2} A_{z}\right),
$$

where $k=\omega \sqrt{\varepsilon_{0} \mu_{0}}$ is free space constant and $A_{z}$ is the axial component of the magnetic vector potential given by [5]

$$
A_{z}=\frac{\mu}{4 \pi} \int_{-L}^{L} g_{E}\left(z, z^{\prime}\right) I\left(z^{\prime}\right) d z^{\prime},
$$

where $\mu$ is free space permittivity, $I\left(z^{\prime}\right)$ is induced axial current, and $g_{E}\left(z, z^{\prime}\right)$ is the so-called exact Green function for the thick cylinder [5]:

$$
g_{E}\left(z, z^{\prime}\right)=\frac{1}{2 \pi} \int_{0}^{2 \pi} \frac{1}{R} d \varphi,
$$

in which $R$ is the distance between source point $z^{\prime}$ and observation point $z$, both of them located on thick wire surface [5]:

$$
R=\sqrt{\left(z-z^{\prime}\right)^{2}+\left(2 a \sin \frac{\varphi}{2}\right)^{2}} .
$$

Combining (1)-(7) and after some mathematical manipulation, Pocklington's integrodifferential equation for imperfectly conducting wire is obtained [5]:

$$
\begin{aligned}
E_{z}^{\mathrm{inc}}= & \frac{1}{j 4 \pi \omega \varepsilon_{0}} \int_{-L}^{L}\left[\frac{\partial^{2}}{\partial z^{2}}+k^{2}\right] g_{E}\left(z, z^{\prime}\right) I\left(z^{\prime}\right) d z^{\prime} \\
& +I(z) Z_{L}(z) .
\end{aligned}
$$

The conducting and dielectric properties of the human body are taken into account in terms of impedance $Z_{L}(z)$ $[1,2,5]$ :

$$
Z_{L}(z)=\frac{1}{a^{2} \pi(\sigma+j \omega \varepsilon)}+\frac{Z_{L 0}}{2 L} .
$$




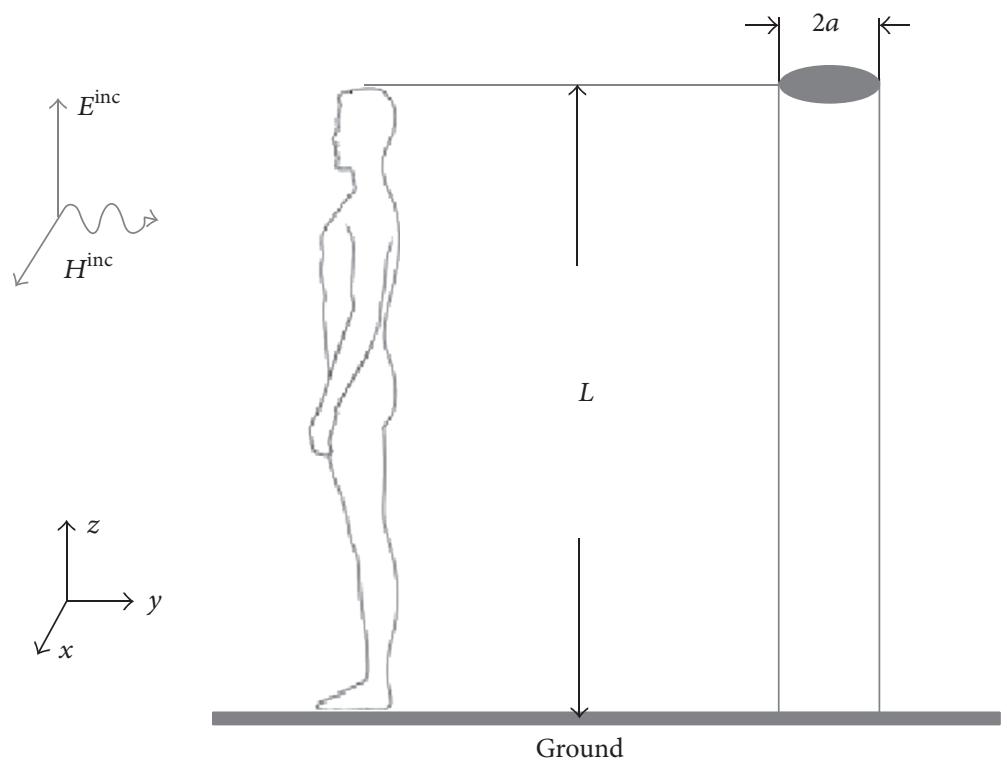

FIGURE 1: Cylindrical model of the human body exposed to electromagnetic field.

Analytical and numerical solutions of integral equation (8) are outlined in the following subsections, respectively.

Once the axial current is determined, it is possible to calculate current density, electric field, and other related parameters [5], such as the current density

$$
J(z)=\frac{I(z)}{a^{2} \pi}
$$

and the induced electric field

$$
E^{\mathrm{int}}(z)=\frac{J(z)}{\sigma}
$$

2.2. Analytical Solution. Equation (8) can be solved analytically according to the procedure presented in $[1,2]$ and the solution is given by

$$
\begin{array}{r}
I\left(z^{\prime}\right)=\frac{j k 2 \pi L E_{z}^{\mathrm{inc}}}{\zeta_{0} \Psi}\left[1-\frac{z^{2}}{L^{2}}-\frac{Z_{L 0}}{Z_{0}+Z_{L 0}}\left(1-\frac{z}{L}\right)\right], \\
-L<z<L,
\end{array}
$$

where $\zeta_{0}=120 \pi \Omega$ is the free space impedance, $Z_{L 0}=1 / j \omega C$, $C$ is the capacity between the soles of the feet and their image in the earth, and $\Psi=\Psi\left(z_{\max }\right)$ is a function depending on the position of maximum current $[1,2]$.

Since this paper deals with the case when man is standing barefoot on PEC ground, then $Z_{L 0}=0$ (the body is well grounded) and (12) simplifies into $[1,2]$

$$
I\left(z^{\prime}\right)=\frac{j k 2 \pi L E_{z}^{\mathrm{inc}}}{\zeta_{0} \Psi}\left[1-\frac{z^{2}}{L^{2}}\right], \quad-L<z<L
$$

where $\Psi=2 \ln (L / a)-3[1,2]$.
2.3. Numerical Solution. Numerical solution of (8) has been presented in [5], and it is briefly outlined here for the sake of completeness. Applying the GB-BEM to (8) results in the following matrix equation:

$$
\sum_{i=1}[Z]_{j i}\{I\}_{i}=\{V\}_{j}, \quad j=1,2, \ldots, M,
$$

where $[Z]_{j i}$ is mutual impedance matrix, $\{I\}_{i}$ contains unknown axial current coefficients, and finally $\{V\}_{j}$ is vector containing excitation function [5]:

$$
\begin{aligned}
& {[Z]_{j i}=\frac{1}{j 4 \pi \omega \varepsilon_{0}}\left(\int_{\Delta_{j}}\{D\}_{j} \int_{\Delta_{i}}\left\{D^{\prime}\right\}_{i}^{T} g_{E}\left(z, z^{\prime}\right) d z^{\prime} d z\right.} \\
& \left.\quad+k^{2} \int_{\Delta_{j}}\{f\}_{j} \int_{\Delta_{i}}\left\{f^{\prime}\right\}_{i}^{T} g_{E}\left(z, z^{\prime}\right) d z^{\prime} d z\right) \\
& \quad+\int_{\Delta_{i}} Z_{L}(z)\{f\}_{j}\{f\}_{i}^{T} d z, \\
& \{V\}_{j}=\int_{\Delta l_{j}} E_{z}^{\text {inc }}\{f\}_{j} d z .
\end{aligned}
$$

In the range of extremely low frequencies, the incident electric field is assumed to be constant over the body and equal to $E_{0}$ so the local voltage corresponds to [5]

$$
\{V\}_{j}=+\frac{E_{0}}{2} \Delta l\left\{\begin{array}{l}
1 \\
1
\end{array}\right\} .
$$

Vector $\{f\}_{j}$ contains linear shape functions and $\{D\}_{j}$ their derivatives. Shape functions are given in the form of Lagrange's polynomials [5].

\section{Stochastic Model}

Integrating stochastic analysis into presented deterministic model statistical margins could be determined for variations 
of input data. The goal is to propagate uncertainties from the input to the output and calculate statistical moments to assess confidence intervals. Various stochastic techniques have been developed in the past decade such as unscented transform (UT), stochastic collocation (SC), polynomial chaos expansion (PCE) method, and Kriging technique. These methods were successfully applied in various electromagnetic compatibility problems [16-18].

Comparing stochastic collocation to well established and widely accepted Monte Carlo methods, the main advantage of SC over MC would be simplicity and less computational cost. On the other hand, MC methods are more convenient in case of large number of input random variables [19]. Also, SC method is nonintrusive; that is, it is not necessary to change existing deterministic code which is an advantage compared to the more accurate but at the same time more complicated and intrusive polynomial chaos expansion method [19].

Theoretical background of SC method can be found elsewhere [16-19], but, for the sake of completeness, some basics are given here, as well.

3.1. Fundamentals of Stochastic Collocation. The fundamental principle of SC technique lies in polynomial approximation of the considered output $E$ for $N$ given random parameters. Random parameter $Z$ is defined as follows $[16,17]$ :

$$
Z=Z^{0}+\widehat{u}
$$

where $Z^{0}$ is the initial (mean) value and $\widehat{u}$ is the random variable $(\mathrm{RV})$ with a priori chosen statistical distribution.

Function $t \rightarrow E\left(Z^{0}, t\right)$ is expanded over a stochastic space using the Lagrangian basis functions [16, 17]:

$$
E\left(Z^{0} ; t\right)=\sum_{i=1}^{n} E_{i}\left(Z^{0}\right) L_{i}(t)
$$

where

$$
L_{i}(t)=\prod_{j=1, j \neq i}^{n} \frac{\left(t-t_{j}\right)}{\left(t_{j}-t_{i}\right)}
$$

is $(n-1)$ degree Lagrange polynomial.

An interesting property of the Lagrangian basis is

$$
L_{i}\left(t_{j}\right)=\delta_{i j}
$$

so that

$$
E_{i}\left(Z^{0}\right)=E\left(Z^{0} ; t_{i}\right) .
$$

Following the definition from statistics for the mean, the expected value of output $E$ of interest can be calculated as

$$
\begin{aligned}
\left\langle E\left(Z^{0} ; t\right)\right\rangle & =\int_{D} E\left(Z^{0} ; t\right) \operatorname{pdf}(t) d t \\
& =\int_{D} \sum_{i=1}^{n} E_{i}\left(Z^{0}\right) L_{i}(t) \operatorname{pdf}(t) d t \\
& =\sum_{i=1}^{n} E_{i}\left(Z^{0}\right) \int_{D} L_{i}(t) \operatorname{pdf}(t) d t .
\end{aligned}
$$

The final form of (22) can be written as

$$
\left\langle E\left(Z^{0} ; t\right)\right\rangle=\sum_{i=1}^{n} E_{i}\left(Z^{0}\right) \omega_{i}
$$

where weight $\omega_{i}$ is given as

$$
\omega_{i}=\int_{D} L_{i}(t) \operatorname{pdf}(t) d t .
$$

Note that function $\operatorname{pdf}(t)$ is the probability density function of RV $\widehat{u}$ from (17).

The order $(n-1)$ of approximation depends on the number of chosen $t_{i}$ points (sigma points). The computation of integral in (24) is based on Gaussian quadrature with identical sigma points. The convergence is dependent on the number of chosen sigma points.

This overview is given for the case of single RV, but it can be easily extended to the case of $N$ RVs. For example, if $N=2$, then

$$
\begin{aligned}
& Z=Z^{0}+\widehat{u}_{Z}, \\
& Y=Y^{0}+\widehat{u}_{Y} .
\end{aligned}
$$

Function $(s, t) \rightarrow E_{i j}\left(Y^{0}, Z^{0} ; s, t\right)$ can be projected on a Lagrangian basis:

$$
E_{i j}\left(Y^{0}, Z^{0} ; s, t\right)=\sum_{i=1}^{n} \sum_{j=1}^{n} E_{i j}\left(Y^{0}, Z^{0}\right) L_{i}(s) L_{j}(t) .
$$

The mean is given by

$$
\left\langle E\left(Y^{0}, Z^{0} ; s, t\right)\right\rangle=\sum_{i=1}^{n} \sum_{j=1}^{n} E_{i j}\left(Y^{0}, Z^{0}\right) \omega_{i} \omega_{j}
$$

and weights are computed from relation

$$
\begin{aligned}
& \omega_{i}=\int_{D} L_{i}(s) \operatorname{pdf}(s) d s, \\
& \omega_{j}=\int_{D} L_{j}(t) \operatorname{pdf}(t) d t .
\end{aligned}
$$

Furthermore, $N$-dimensional problems can be solved using tensor product in each direction. However, for very large $N$, stochastic collocation would become complex for practical use.

In addition to the mean, other higher statistical moments can be readily computed and the expressions are given in Table 1 for one-dimensional RV case. An extension to $\mathrm{N}$ dimensional problems is straightforward.

\section{Computational Examples}

First, in Section 4.1, univariate examples are given, while Section 4.2 deals with the case of three input RVs. Note that uncertain inputs are integrated in both analytical and numerical deterministic solutions. 
TABLE 1: SC computation of high-order statistical outputs for the one-RV case (random output $E$ ).

\begin{tabular}{lcc}
\hline $\begin{array}{l}\text { Statistical } \\
\text { moment }\end{array}$ & Order $p$ & $\begin{array}{c}\text { Computation SC }(n-1) \\
\text { order }\end{array}$ \\
\hline Mean & 1 & $\langle E\rangle=\sum_{i=1}^{n} \omega_{i} E_{i}$ \\
Variance & 2 & $\sigma_{E}^{2}=\sum_{i=0}^{n} \omega_{i} E_{i}^{2}-\langle E\rangle^{2}$ \\
Skewness & 3 & $\operatorname{skew}(E)=\sum_{i=1}^{n} \frac{\left|\omega_{i} E_{i}-\langle E\rangle\right|^{3}}{\left|\sigma_{E}^{2}\right|^{3 / 2}}$ \\
Kurtosis & 4 & $\operatorname{kurt}(E)=\sum_{i=1}^{n} \frac{\left|\omega_{i} E_{i}-\langle E\rangle\right|^{4}}{\left|\sigma_{E}^{2}\right|^{2}}$ \\
\hline
\end{tabular}

TABLE 2: Random input variables.

\begin{tabular}{lccc}
\hline & $\begin{array}{c}\text { Initial (mean) } \\
\text { value }\end{array}$ & $\begin{array}{c}\text { Random } \\
\text { variable }\end{array}$ & Distribution \\
\hline Body length $(L)$ & $L^{0}=1.75 \mathrm{~m}$ & $\widehat{u}_{L}$ & $\bigcup[-2,2] \mathrm{cm}$ \\
Body radius $(a)$ & $a^{0}=0.14 \mathrm{~m}$ & $\widehat{u}_{a}$ & $\bigcup[-2,2] \mathrm{cm}$ \\
Conductivity $(\sigma)$ & $\sigma^{0}=0.5 \mathrm{~S} / \mathrm{m}$ & $\widehat{u}_{\sigma}$ & $\bigcup[-0.1,0.1] \mathrm{S} / \mathrm{m}$ \\
\hline
\end{tabular}

$\mathrm{U}$ indicates that the random variable is uniformly distributed.

Input variables chosen as RVs are body length, radius, and conductivity, respectively. Random inputs are given in Table 2 and all RVs follow uniform distribution. In principle, stochastic studies are carried out in two steps: first to statistically model input data and then to solve stochastic numerical or analytical model, respectively [16]. The mean values for each RV are given in literature $[1,2,5]$.

Sigma-weight pairs for each of the random variables are given in Table 3. Convergence is accomplished for three sigma points in all examples and therefore there is no need to increase the number of points.

The results for all scenarios are presented in the following way: distribution of the mean value for the output of interest, which is either induced current or internal electric field, is given along the human body starting from the feet to the top of the head. Also, figures display the confidence interval around the mean value given as mean value \pm 3 or \pm 2 standard deviations.

4.1. Univariate Examples. The first example selected is the human body exposed to incident electric field $E_{z}^{\text {inc }}=10^{4} \mathrm{~V} / \mathrm{m}$ at frequency of $60 \mathrm{~Hz}$ with body length $L$ as RV. Then, at the second example, radius $a$ is chosen as input RV and finally conductivity $\sigma$ (Table 2). The results are presented in Figures $2-4$, respectively. Stochastic analysis is carried out for both analytical and numerical deterministic solutions.

Figures 2-4 show the highest variability of the results in the case when the radius of the body is taken as RV input (Figure 4). The results are practically nondependent on conductivity (Figure 2) which is in accordance with the analytic prediction; that is, the current does not vary with the change in conductivity $[1,2]$. Taking into account the influence of each RV on the output, it is possible to use SC for sensitivity analysis to exclude the less significant variables

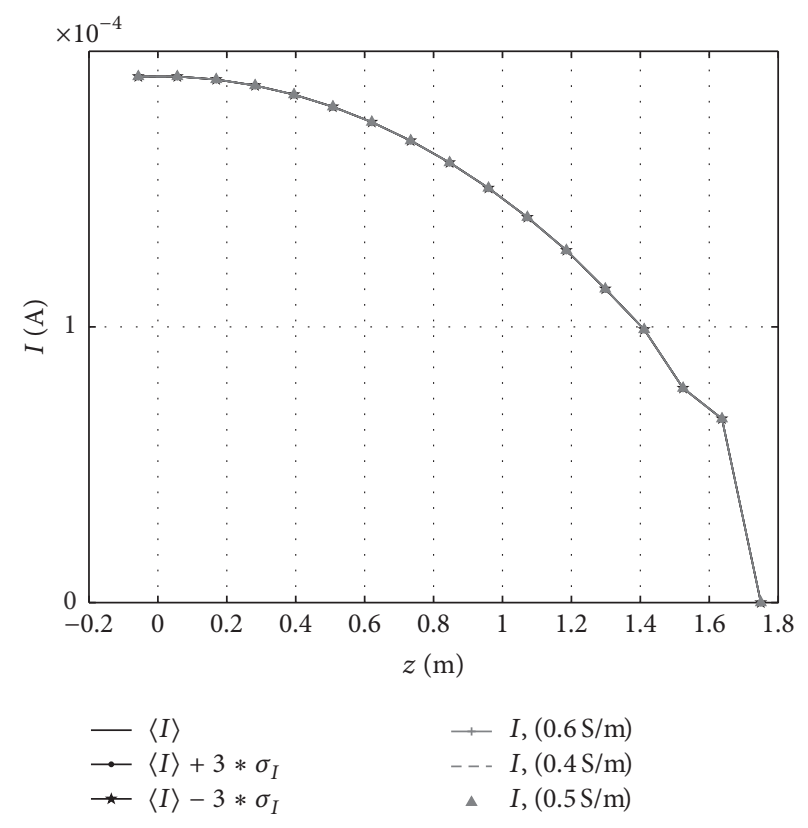

FIGURE 2: Induced axial current in the human body with conductivity $\sigma$ as RV: $\langle I\rangle$ is stochastic expected value, $\sigma_{I}$ is standard deviation, and $I$ is the value obtained from numerical deterministic model.

TABLE 3: Sigma-weight pairs for each of the random variables.

\begin{tabular}{cccc}
\hline$N$ & PT1 & PT2 & PT3 \\
\hline$L(\mathrm{~m})$ & & & \\
$S_{i}$ & 1.7345 & 1.7500 & 1.7655 \\
$\omega_{i}$ & 0.2778 & 0.4444 & 0.2778 \\
$a(\mathrm{~m})$ & & & \\
$S_{i}$ & 0.1245 & 0.1400 & 0.1555 \\
$\omega_{i}$ & 0.2778 & 0.4444 & 0.2778 \\
$\sigma(\mathrm{S} / \mathrm{m})$ & & & \\
$S_{i}$ & 0.4225 & 0.5000 & 0.5775 \\
$\omega_{i}$ & 0.2778 & 0.4444 & 0.2778 \\
\hline
\end{tabular}

from observation for case when the number of random input variables grows high.

An additional task is to assess the confidence margins for the output of interest. According to the usual procedure to define confidence interval as mean value \pm 3 standard deviations, it is demonstrated that the extreme values are not sufficient to predict the dispersion of results around mean trend.

4.2. Multivariate Examples. In this section, the example with all three input random variables is considered. Sigma/weight pairs are given in Table 3 . The human body is illuminated by incident field $E_{z}^{\text {inc }}=10^{4} \mathrm{~V} / \mathrm{m}$ at frequency $f=60 \mathrm{~Hz}$. The results in Figure 5 are very similar to the ones in Figure 4 where only radius is considered as random variable, thus verifying the variability of the output current to mostly depend on variability of radius that belongs to particular cylindrical representation of the human body. Though this conclusion is related to the case of uniform distribution 


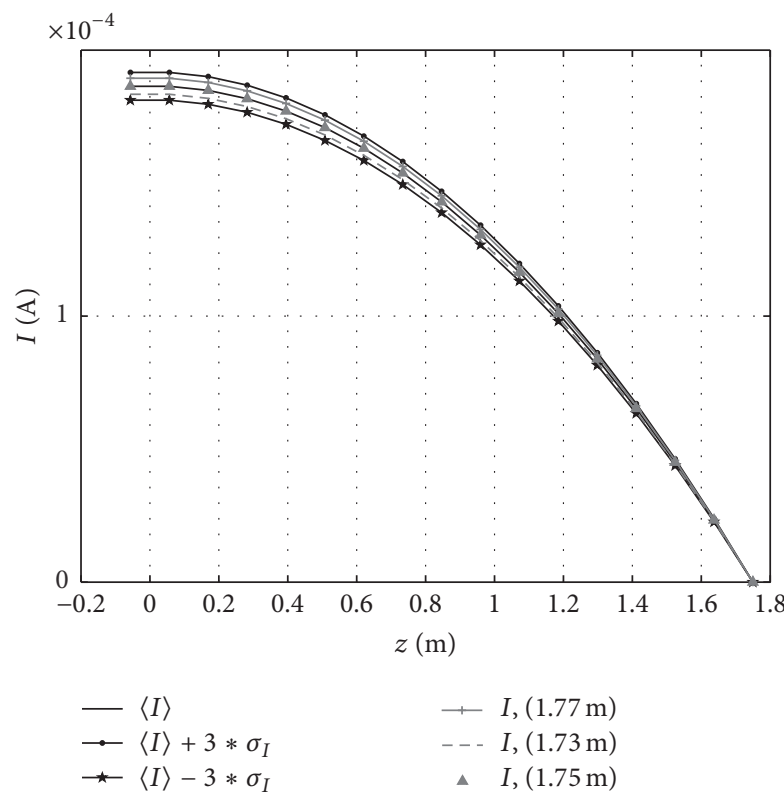

(a)

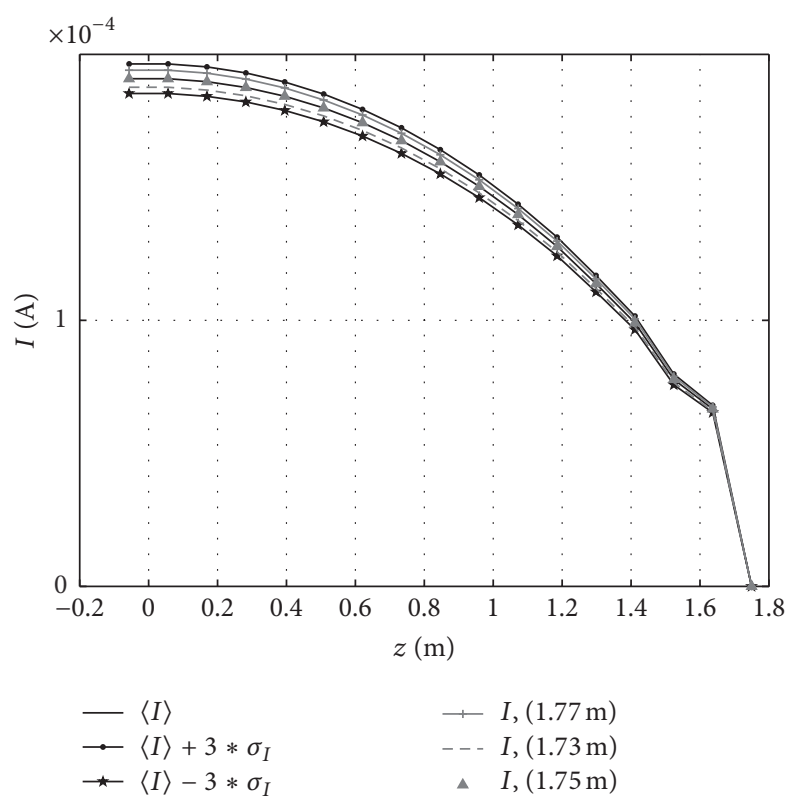

(b)

FIGURE 3: Induced axial current in the human body with length $L$ as RV: $\langle I\rangle$ is stochastic expected value, $\sigma_{I}$ is standard deviation, and $I$ is the value obtained from analytical (a) and numerical deterministic model (b).

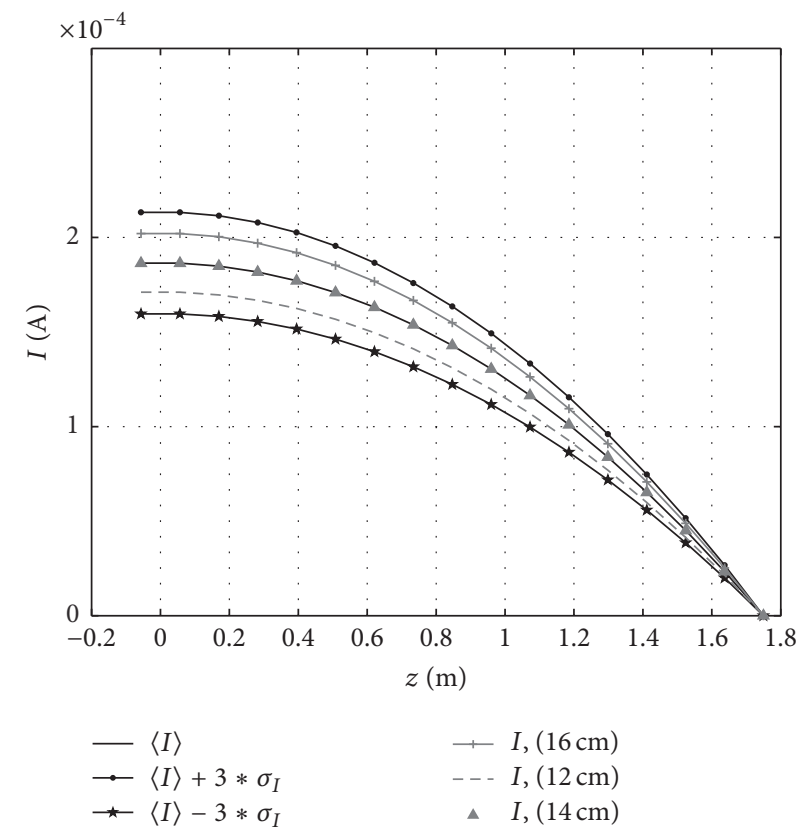

(a)

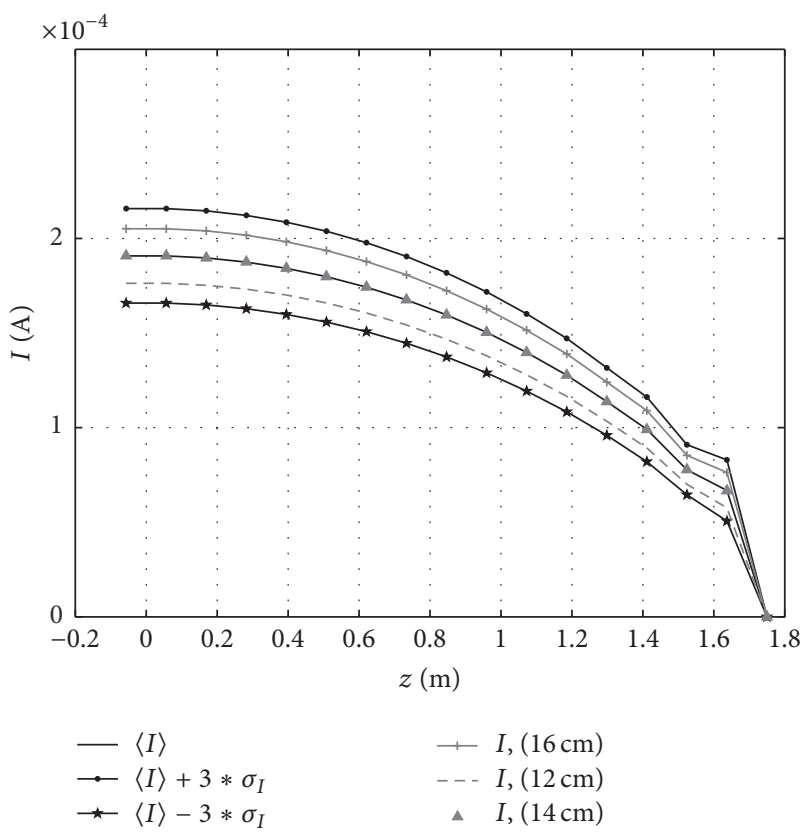

(b)

FIGURE 4: Induced axial current in the human body with radius $a$ as RV: $\langle I\rangle$ is stochastic expected value, $\sigma_{I}$ is standard deviation, and $I$ is the value obtained from analytical (a) and numerical deterministic model (b).

of random variables, the computation is also applicable to different distributions of random variables.

It is worth noting that the results represented in Figures 2-5 show the distribution of the induced current along the human body starting from the feet to the top of the head.
This is consistent with ICNIRP's basic restriction on exposure of humans to ELF electric fields from 1998 which is induced current density [13].

However, international standards [14, 15], also recommended by WHO, report the change in the basic restriction 


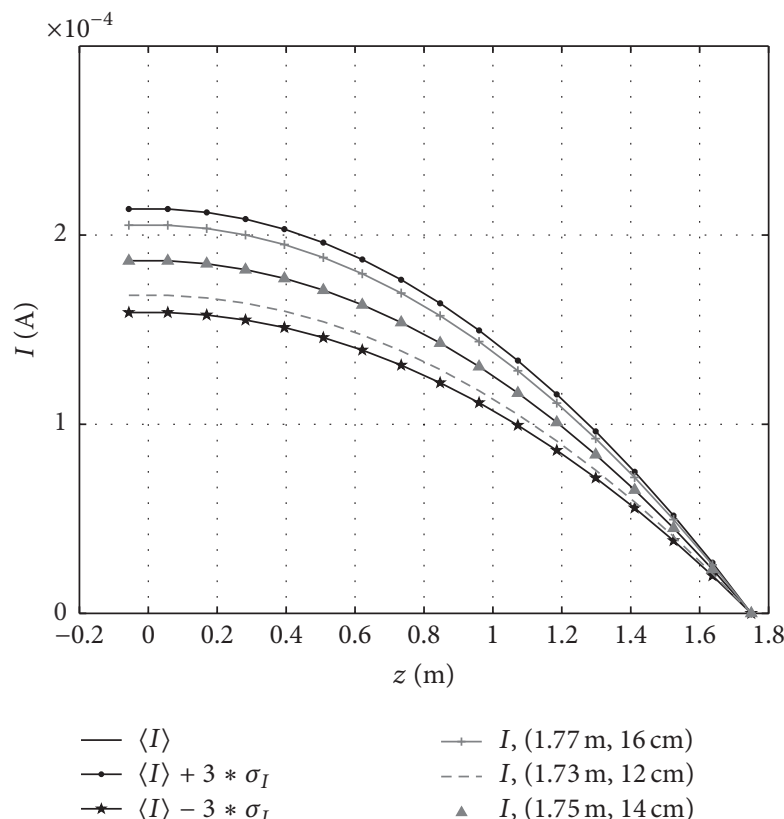

(a)

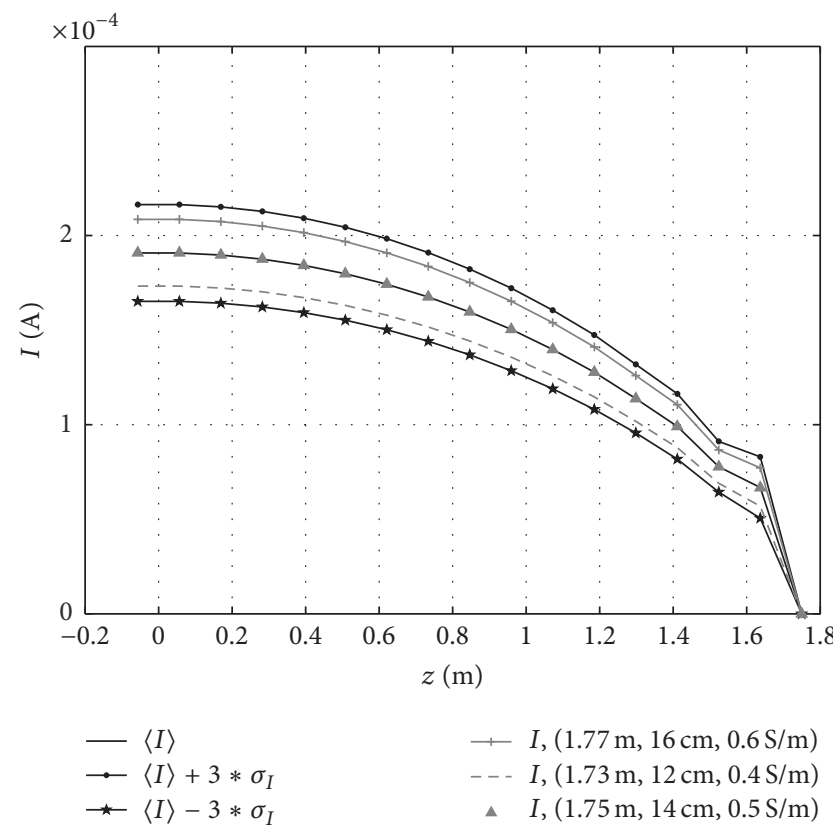

(b)

FIGURE 5: Induced axial current in the human body: $\langle I\rangle$ is stochastic expected value, $\sigma_{I}$ is standard deviation, and $I$ is the value obtained from (a) analytical deterministic model, two input RVs: length and radius $(L, a)$, and (b) numerical deterministic model, three input RVs: length, radius, and conductivity $(L, a, \sigma)$.

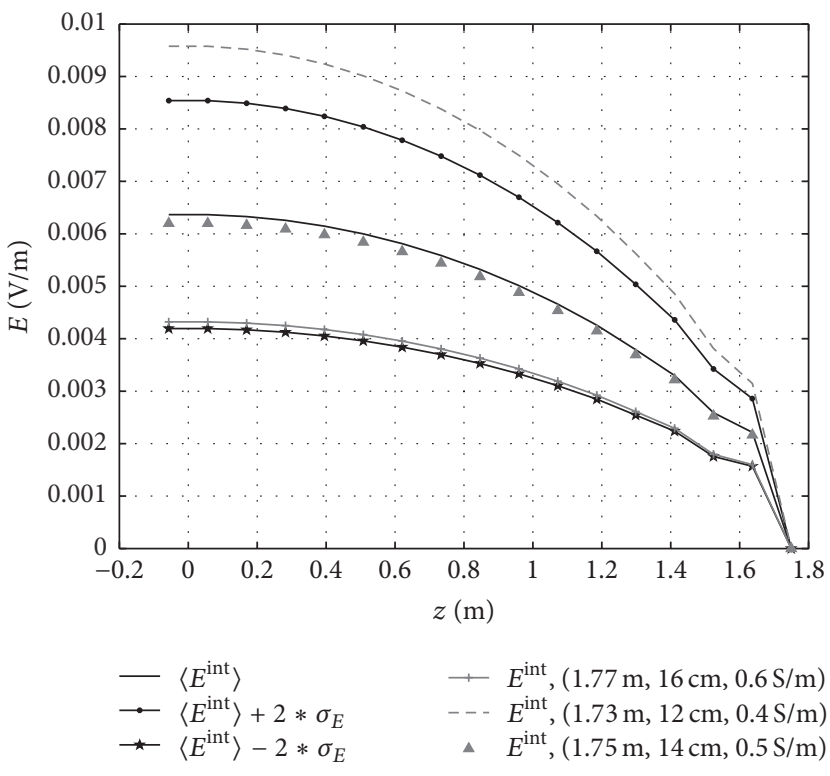

(a)

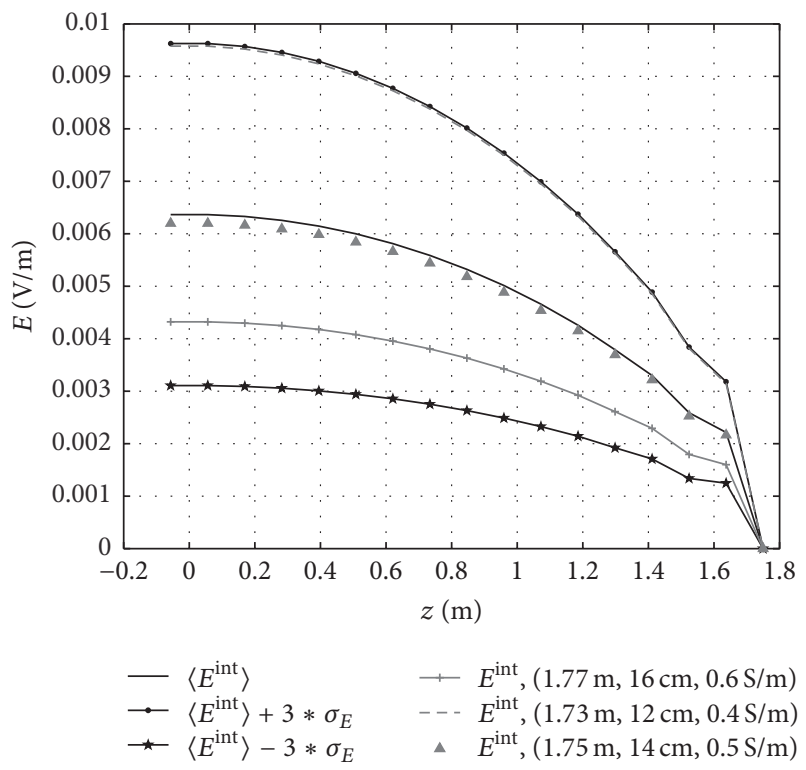

(b)

FIGURE 6: Induced electric field in the human body with three RVs-length, radius, and conductivity $(L, a, \sigma):\left\langle E^{\text {int }}\right\rangle$ is stochastic expected value, $\sigma_{E}$ is standard deviation, and $E^{\text {int }}$ is the value obtained from numerical deterministic model. (a) Confidence interval of $95.45 \%$ is given as $\pm 2 \sigma_{E}$ margins around mean. (b) Confidence interval of $99.7 \%$ is given as $\pm 3 \sigma_{E}$ margins around mean.

parameter on ELF electric field exposure from induced current into induced electric field inside the body $\vec{E}^{\text {int }}$. Therefore, in Figure 6, the mean trend of the induced electric field obtained from (11) is represented along with the confidence intervals: the mean value \pm 2 standard deviations for $95.45 \%$ and the mean value \pm 3 standard deviations for $99.7 \%$ certainty of coverage (Figures 6(a) and 6(b), resp.). The extreme values are completely included inside the confidence margins for the case of $99.7 \%$ coverage, while, in $95.45 \%$ cases, upper extreme value is not likely to happen. Once again, 
it is demonstrated that extreme values are not sufficient to predict the dispersion of results around mean trend, since lower extreme values are much higher than the lower interval margin in case of $99.7 \%$ coverage. Also, the stochastically computed mean is higher than the deterministic one.

\section{Conclusion}

The paper deals with the application of stochastic collocation (SC) method in modelling the human body exposed to LF fields using the cylindrical antenna representation of the body. The deterministic model has already been presented and the new perspective has been given in this work. Taking into account the constant interest of public in potentially adverse health effects of ubiquitous EM radiation, this paper extends the analysis from the stochastic point of view. Presented examples provide the mean value for induced current and induced electrical field with the confidence margins taking into account a priori determined variability of input parameters. It is demonstrated that extreme values of input RVs are not sufficient to predict the dispersion of the output around mean trend. Taking into account confidence intervals obtained from stochastic models, basic restrictions in EM dosimetry can be established with better precision.

\section{Competing Interests}

The authors declare that they have no competing interests.

\section{References}

[1] R. W. P. King, "Fields and currents in the organs of the human body when exposed to power lines and VLF transmitters," IEEE Transactions on Biomedical Engineering, vol. 45, no. 4, pp. 520530, 1998.

[2] R. W. P. King, "A review of analytically determined electric fields and curents induced in the human body when exposed to 5060-Hz electromagnetic fields," IEEE Transactions on Antennas and Propagation, vol. 52, no. 5, pp. 1186-1192, 2004.

[3] B. Kibret, A. K. Teshome, and D. T. H. Lai, "Characterizing the human body as a monopole antenna," IEEE Transactions on Antennas and Propagation, vol. 63, no. 10, pp. 4384-4392, 2015.

[4] B. Kibret, A. K. Teshome, and D. T. H. Lai, "Cylindrical antenna theory for the analysis of whole-body averaged specific absorption rate," IEEE Transactions on Antennas and Propagation, vol. 63, no. 11, pp. 5224-5229, 2015.

[5] D. Poljak and Y. F. Rashed, "The boundary element modelling of the human body exposed to the ELF electromagnetic fields," Engineering Analysis with Boundary Elements, vol. 26, no. 10, pp. 871-875, 2002.

[6] R. W. P. King and S. S. Sandler, "Electric fields and currents induced in organs of the human body when exposed to ELF and VLF electromagnetic fields," Radio Science, vol. 31, no. 5, pp. 1153-1167, 1996.

[7] O. P. Gandhi and J. Y. Chen, "Numerical dosimetry at powerline frequencies using anatomically based models," Bioelectromagnet, supplement 1, pp. 43-60, 1992.

[8] R. J. Spiegel, "Numerical determination of induced currents in humans and baboons exposed to $60-\mathrm{Hz}$ electric fields," IEEE
Transactions on Electromagnetic Compatibility, vol. 23, no. 4, pp. 382-390, 1981.

[9] A. Chiba, K. Isaka, Y. Yokoi, M. Nagata, and M. Kitagawa, "Application of finite element method to analysis of induced current densities inside human model exposed to $60 \mathrm{~Hz}$ electric field," IEEE Transactions on Power Apparatus and Systems, vol. 103, no. 7, pp. 1895-1902, 1984.

[10] W. T. Kaune and F. A. McCreary, "Numerical calculation and measurement of $60-\mathrm{Hz}$ current densities induced in an upright grounded cylinder," Bioelectromagnetics, vol. 6, no. 3, pp. 209220, 1985.

[11] J. P. Reilly and A. Hirata, "Low-frequency electrical dosimetry: research agenda of the IEEE International Committee on Electromagnetic Safety," Physics in Medicine and Biology, vol. 61, no. 12, pp. R138-R149, 2016.

[12] I. Magne and F. Deschamps, "Electric field induced in the human body by uniform $50 \mathrm{~Hz}$ electric or magnetic fields: bibliography analysis and method for conservatively deriving measurable limits," Journal of Radiological Protection, vol. 36, no. 3, pp. 419-436, 2016.

[13] ICNIRP, "ICNIRP guidelines for limiting exposure to timevarying electric, magnetic fields and electromagnetic fields (up to $300 \mathrm{GHz}$ )," Health Physics, vol. 74, no. 4, pp. 494-522, 1998.

[14] International Commission on Non-Ionizing Radiation Protection (ICNIRP), "Guidelines for limiting exposure to timevarying electric and magnetic fields $(1 \mathrm{~Hz}-100 \mathrm{kHz})$," Health Physics, vol. 99, no. 6, pp. 818-836, 2010.

[15] IEEE Std C95.6 ${ }^{\mathrm{TM}}$, IEEE Standard for Safety Levels with Respect to Human Exposure to Electromagnetic Fields, $0-3 \mathrm{kHz}$, The Institute of Electrical and Electronics Engineers, New York, NY, USA, 2002.

[16] S. Lalléchère, P. Bonnet, and F. Paladian, "Electrical stochastic modelling of cell for bio-electromagnetic compatibility applications," Annals of Telecommunications, vol. 69, no. 5, pp. 295-308, 2014.

[17] H. Dodig, S. Lalléchère, P. Bonnet, D. Poljak, and K. El Khamlichi Drissi, "Stochastic sensitivity of the electromagnetic distributions inside a human eye modeled with a 3D hybrid BEM/FEM edge element method," Engineering Analysis with Boundary Elements, vol. 49, pp. 48-62, 2014.

[18] S. Lalléchère, S. Antonijevic, K. El Khamlichi Drissi, and D. Poljak, "Optimized numerical models of thin wire above an imperfect and lossy ground for GPR statistics," in Proceedings of the 17th International Conference on Electromagnetics in Advanced Applications (ICEAA '15), pp. 907-910, Torino, Italy, September 2015.

[19] D. Xiu, "Fast numerical methods for stochastic computations: a review," Communications in Computational Physics, vol. 5, no. 2-4, pp. 242-272, 2009. 


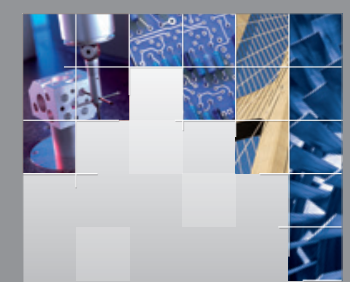

\section{Enfincering}
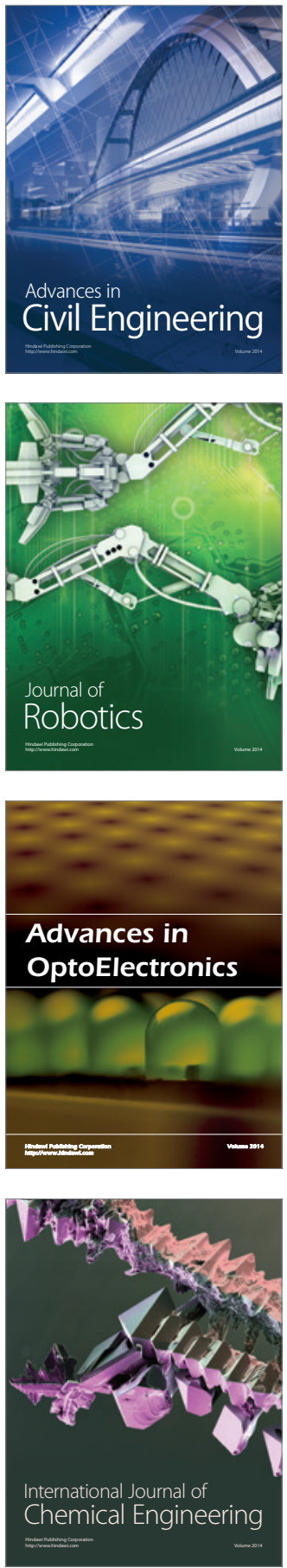

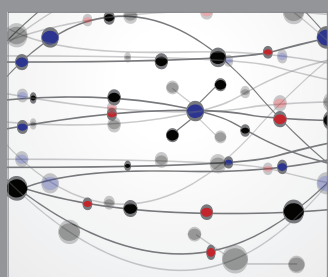

The Scientific World Journal

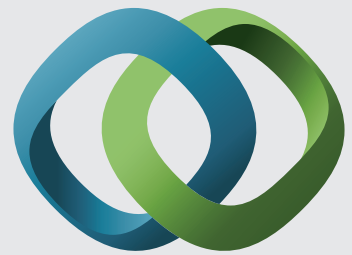

\section{Hindawi}

Submit your manuscripts at

http://www.hindawi.com
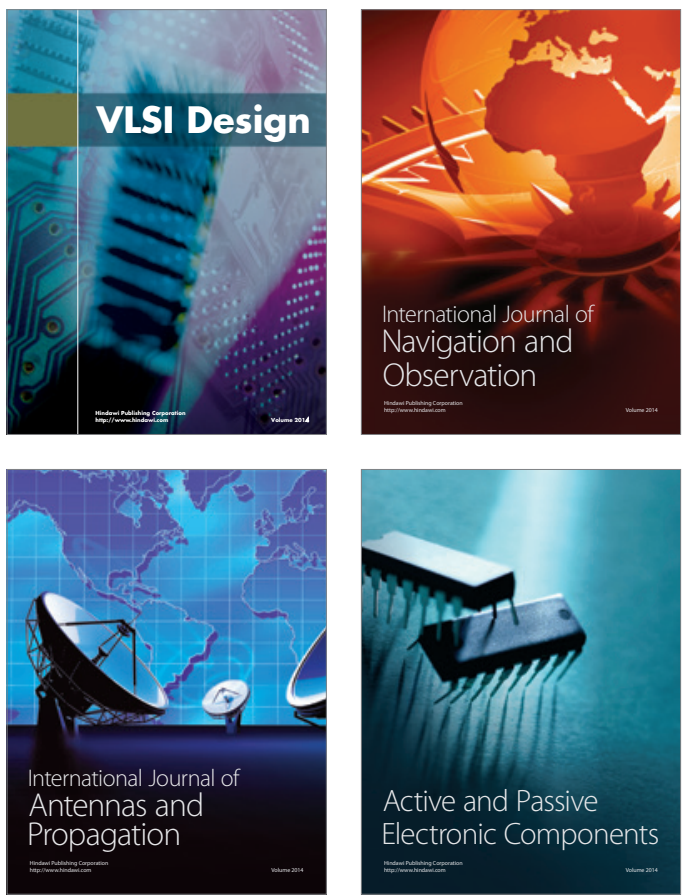
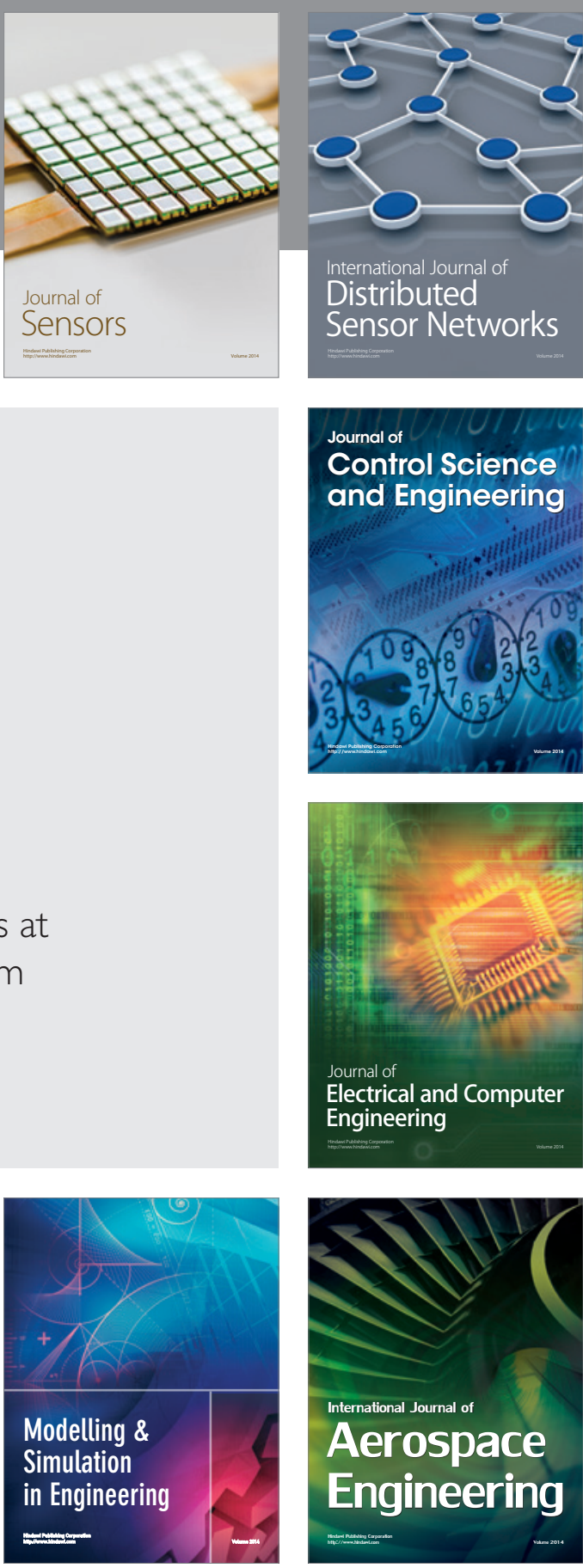

International Journal of

Distributed

Sensor Networks

Journal of

Control Science

and Engineering
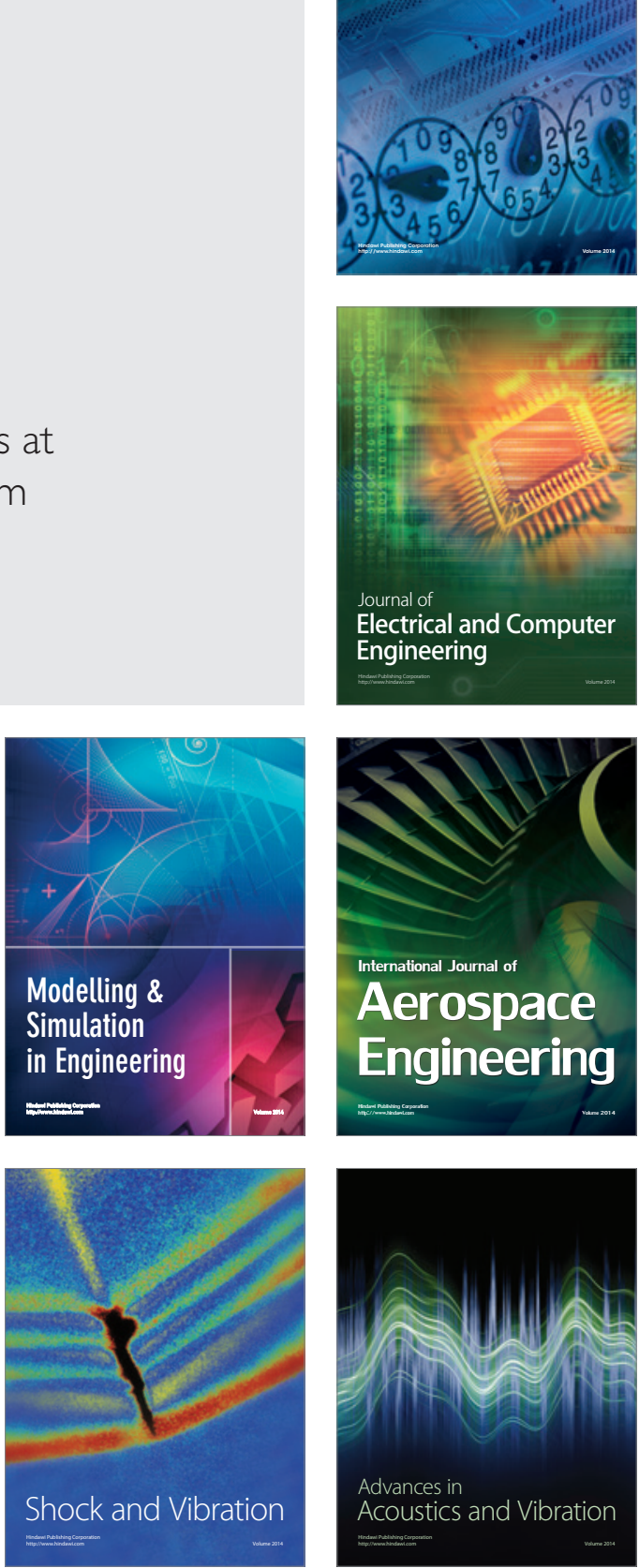